\title{
Treatment of Difficult Case of Valgus Osteoarthritis Knee
}

\author{
Hazim Sakalla* \\ Sultan Bin Abdulaziz Humanitarian City, Saudi Arabia
}

*Corresponding author : Hazim Sakalla, Orthopedic Surgeon at Sultan Bin

Abdulaziz Humanitarian City, Saudi Arabia.

Received Date: December 13, 2018

Published Date: January 02, 2019

\section{Clinical Image}

59 years old female who came to the Clinic complaining of severe knee pain which affecting her activity, she has valgus knee deformity (knock knee) her valgus knee was about 25 degree and she was seen by many Orthopedic doctors who kept pushing away the patient till she found us. She has DM and HTN.

After I discussed the case with the patient and she was agreed to proceed with bilateral total knee replacement. The patient was sent for clearance and after clearance she was taken to theater and we performed bilateral TKR at the same time, above is the x-ray preoperative on the left and post op on the right which showed the severe deformity and how it was corrected with soft tissue balancing technique (lateral soft tissue release) (Figure 1).

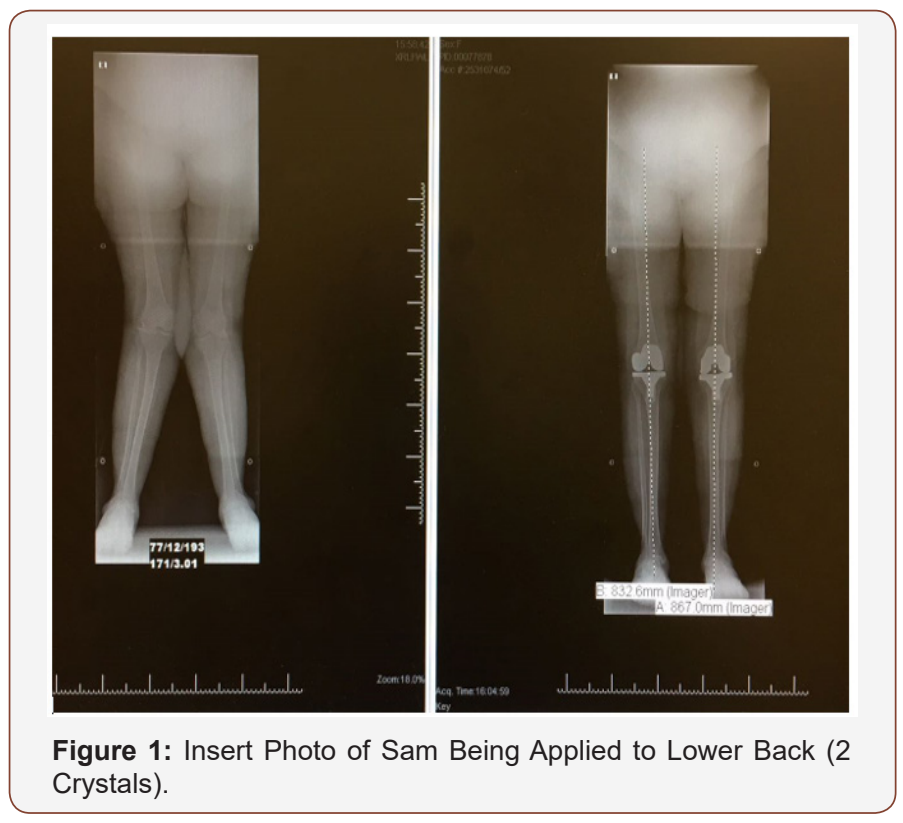

\section{Steps of Release}

\section{Varus release in-order}

1. Remove medial osteophytes from proximal tibia.
2. Medial soft tissue release in order.

- Deep MCL .

- Postmedial capsule(posterior capsule )and PCL.

- Semimembranus.

- $\quad$ Superficial MCL subperiosteally

- Pes anserinus

- Medial head of gastrocnemius.

Valgus release in order

1. ITB from gerdys tubercle.

2. lateral capsular attachment to the tibia

3. Posterolatiral capsule+/- PCL.

4. Popliteus tendon.

5. LCL.

6. lateral head of gastrocnemius.

7. lateral intramascular septum with vastus lateralis +/biceps tendon.

\section{Flexion release}

1. More proximal femoral bone resection.

2. Remove posterior osteophytes.

3. Elevation of posterior capsul, release of the PCL.

4. Posterior capsule can be divided transversely near itis origin from the femur with great care.

5. For minimizing bone resection, divide or strip posterior capsular attachment to the femur, which may include both heads of gastrocnemius. 
6. Additional resection from the distal femur undertaken, if unable to attain full extension.

7. If extension restricted, cut more distal femur.

8. If flexsion restricted, cut more posterior femur.

9. If both flexsion and extension restricted cut more proximal tibia.
Acknowledgement

None.

\section{Conflict of Interest}

No Conflict of Interest. 